\title{
Studying Optimal Design of Strict Fractional Frequency Reuse in OFDMA Cellular System
}

\author{
Mahmoud Nagieb, Mona Shokair and Waleed Saad \\ Faculty of Electronic Engineering, \\ El-Menoufia University, \\ Menouf, 32952, Egypt
}

\begin{abstract}
The key characteristic of a cellular network is the ability to reuse frequencies to increase both coverage and capacity. Fractional frequency reuse (FFR) is considered to be an efficient inter-cell interference coordination technique wellsuited for OFDMA based on wireless communication networks where the cells are partitioned into spatial regions with different frequency reuse factors. In this paper, evaluating strict FFR which represents a type of FFR deployments is presented with four different system models by changing the inner-cell shape for each model. System simulations are used to compare and evaluate the effect of changing the inner-cell shape based on strict FFR performance which performed using dense Monte Carlo simulations. In addition, the effects of some system model parameters are discussed.
\end{abstract}

\section{Keywords}

OFDMA, Fractional Frequency Reuse

\section{INTRODUCTION}

Using frequency reuse of one in LTE supports Orthogonal Frequency Reuse (OFDMA) which means that all cells operate on the same frequency channel is to maximize spectral efficiency. Because of the using of OFDMA, intracell users are assumed to be orthogonal to each other and the primary source of interference is inter-cell interference [1]. FFR has been proposed as an ICIC technique in OFDMA based wireless networks that improves network performance [2] [3]. The basic mechanism of FFR corresponds to partitioning the macro-cell service area into spatial regions [4], and each sub-region is assigned with different frequency sub-bands. Therefore, cell edge-zone users do not interfere with center-zone users. As a result, the cell-edge-zone users receive an acceptable signal quality, which subsequently reduces the blocking probability and increases the network capacity. The FFR scheme operates on a relatively large time scale. This is referred to as a static FFR scheme. In contrast, dynamic FFR schemes can operate on short timescales and can be optimized for system utility with varying network dynamics. However, they are more complex and less scalable than static schemes.

There are three FFR schemes presented for OFDMA based networks: strict FFR, soft FFR and FFR3 [5].

Strict FFR is a modification of the traditional frequency reuse used in multi-cell networks. Fig. 1 illustrates a strict FFR deployment with a cell-edge-reuse factor of $\mathrm{N}=3$. Users in each inner cells share the same sub-band frequencies while cell-edge users, bandwidth is partitioned across cells based on reuse factor of N. Strict FFR requires a total of N+1 sub-bands [6].
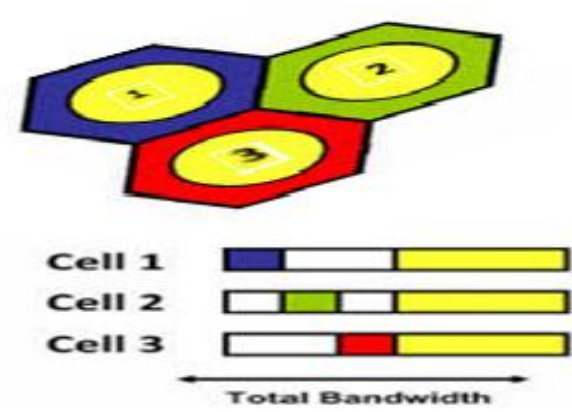

Fig. 1 Strict FFR deployment with $\mathbf{N}=3$ cell edge reuse factor.

Soft FFR uses a cell partitioning technique similar to that of the strict FFR scheme. However, the center zone users are allowed to use the sub-bands of cell-edge zone of the neighboring cells within the cluster. In this scheme, one of the major advantages of soft FFR is that it has better spectrum efficiency than strict FFR .

FFR-3, the macro-cell coverage area is partitioned into center and edge zones, including three sectors each. The entire frequency band is divided into two parts, one part is solely assigned to the center zone and the other part is partitioned into three sub-bands assigned to the three edge zones.

In this paper, the main goals of our strict FFR based LTE network model are reaching to the best design for strict FFR system to maximize throughput, minimize blocking probability and achieve best quality of service at the system. This is done by changing center zone cell shape and obtaining the best performance.

The previous researchers concentrated on the trade-offs associated with strict FFR and soft FFR systems in comparison with universal frequency reuse and sensitivity to the interior radius selection [7]. Also, they focused on optimal design of FFR systems by utilizing advanced techniques such as graph theory [8] and convex optimization [9] [10] to maximize network throughput. No one has studied the performance while changing the inner cell shape which will be focused by this paper.

The rest of the paper is organized as follows, the system model, its specifications and mathematical analysis will be presented in Section 1. Then, Simulation results and its discussion will be investigated in Section 2. Afterwards, the discussions of the simulation results will be discussed in Section 3. Finally, conclusions will be made in Section 4. 


\section{SYSTEM MODEL}

The system model typically assumed for strict FFR over LTE standard. Our model is shown in Fig. 1. It consists of three macro cells in which each cell is partitioned into center and edge zone. The resource allocation between interior and celledge users is proportional to the square of the ratio of interior radius and the cell radius is represented by $R$. this is when user locations are assumed to be uniformly distributed [11]. One of the most important design parameters here is the radius of the center zone of the macro-cell. Using Monte Carlo simulations, it was shown in [5] that, for uniformly users distributions. If the cell-center zone radius (rcenter) is 0.65 times the macro-cell radius $(\mathrm{R})$. The average network throughput is maximized. Therefore, the ratio of ( $\mathrm{rint} / \mathrm{R}$ ) $=0,65$. With Nband total sub-bands available to the cell, the allocation of sub-bands available for the interior users is Nint and exterior users Next is given as,

$$
\begin{aligned}
\mathrm{N}_{\text {int }} & =\left[\mathrm{N}_{\text {band }}\left(\frac{\mathrm{r}_{\text {int }}}{\mathrm{R}}\right)^{2}\right] \\
\mathrm{N}_{\text {ext }} & =\left[\left(\mathrm{N}_{\text {band }}-\mathrm{N}_{\text {int }}\right) / 3\right]
\end{aligned}
$$

In this scheme, the cell-edge users are not interfered with each other, this reduces the inter-cell interference. Also, since the center-zone and edge zone users use different sub-bands, intra-cell interference is mitigated. In contrast, center-zone users will suffer from inter-cell interference because of using the same band.

The associated signal to interference plus noise ratio (SINR) for user $\mathrm{y}$ served by base station $\mathrm{x}$ for strict FFR is given as [7]

$$
\operatorname{SIN}_{\mathrm{y}}=\frac{\mathrm{Ph}_{\mathrm{xy}} \mathrm{G}_{\mathrm{xy}}}{\sigma^{2}+\sum_{\mathrm{z} \in \mathrm{Z}} \mathrm{Ph}_{\mathrm{zy}} \mathrm{G}_{\mathrm{zy}}}
$$

Where $\mathrm{P}$ is the transmit power of the base station, hxy is the exponentially distributed channel fading power, and Gxy is the path loss associated with the channel between user $y$ and base station $\mathrm{x}$, and $\sigma^{2}$ is the noise power. The set $\mathrm{Z}$ represents the entire interfering base stations, i.e. base stations that are using the same sub-band as user $y$. the path-loss factor $G_{x y}$ between a base station and user is based on the simple exponential path-loss expression:

$$
\mathrm{G}_{\mathrm{xy}}=\|\mathrm{x}-\mathrm{y}\|^{-\alpha},
$$

where $\alpha$ is the desired path-loss exponent and equal to 4 [7].

For frequency channel allocation in inner-cell users, SINRth is selected to be the reference for SINRy and SINRy to be compared with it. The decision about the channel frequency assignment is determined by the following, SINRy > SINRth. Then, the frequency channel is selected.

\section{SIMULATION RESULTS \\ 3.1. Simulation Setup}

Several Monte Carlo MATLAB simulation experiments are made to study the performance of strict FFR system models. The simulation parameters are illustrated in Table 1.

\subsection{The impact of the Inner Cell shape}

Four different system models with different inner-cell shapes for strict FFR system (Square (S), Hexagon (H), Circle (C) and Triangle (T)) are shown in Fig. 2. Studying these system models are introduced to obtain the best design for strict FFR system that gives maximum Throughput and minimum blocking probability.

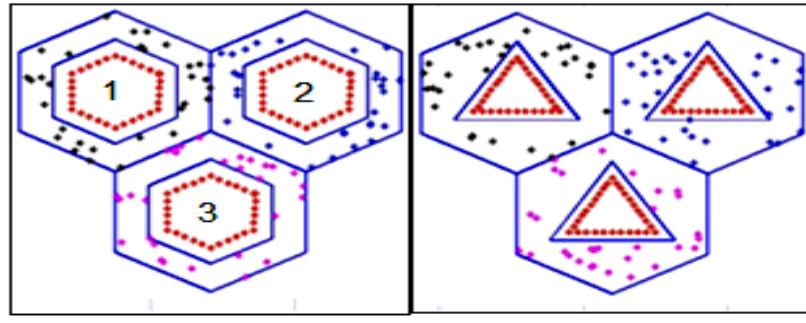

(2.a) Hexagon inner shape

(2.b) Triangle inner shape

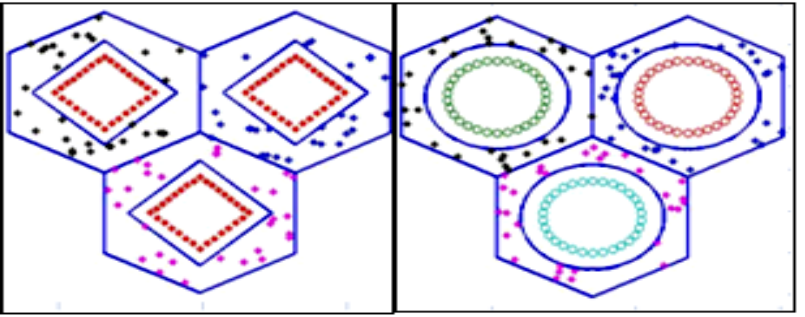

(2.c) Square inner shape (2.d) Circle inner shape

Fig. 2 System model including four different inner-cell shape..

Table 1 System Parameters

\begin{tabular}{|c|c|}
\hline Parameter & Value \\
\hline Outer Cell Radius & $50 \mathrm{~m}$ \\
\hline Inner Cell Radius & $32.5 \mathrm{~m}$ \\
\hline Total Number of Available CH & 50 \\
\hline BS Power & $50 \mathrm{mw}$ \\
\hline Inner Cell Layout & $\begin{array}{c}\text { Square, Hexagon, } \\
\text { Circle \& Triangle }\end{array}$ \\
\hline Background noise (PN) & $-102 \mathrm{dBm}$ \\
\hline User distribution & Uniform \\
\hline Number of users in the system & 180 \\
\hline Simulation Time & $2 * 10^{4} \mathrm{~s}$ \\
\hline Simulation Step time & 0.01 \\
\hline
\end{tabular}

Throughput (THR):is defined as the number of succeed calls over time and can be calculated as below,

$$
\text { THR }=\frac{\text { Success call number }}{\text { simulation time }} .
$$

Blocking probability $(\mathrm{Pb})$ : defined as the probability that a new call request can be denied for lack of resources and can be calculated as below,

$$
\mathrm{Pb}=\frac{\text { block call number }}{\text { total call number }}
$$

The users are distributed randomly in the outer cells. On the contrary in the inner cells, they are allocated to draw the same inner cell shape. That is the way to take the effect of the inner cell shape.

It is worth mention that, the users in cell 1 are shown in Fig. 2 in which have the highest priority followed by the users in cell 2 then cell 3 . This will increase the blocking probability of the cells 2 and 3 compared to cell 1 . 
Fig. 3 ( $a, b$ and c) illustrates the relation between blocking probability of the four models with time in inner-cell number 1,2 and 3 , respectively.

From Fig.3a, it is shown that the triangle and hexagon models give the least blocking probability, while it is shown from Fig. $3 \mathrm{~b}$ that the triangle and square models give the least blocking probability, whilst all models give almost the same blocking probability as shown in Fig. 3c.

From above, the triangle and the hexagon models can be taken as the best shape for inner cell according to the blocking probability of the inner users.

Fig. 3 (d, e and f) shows the relation between blocking probability of the four models with time in outer-cell number 1,2 and 3 , respectively.

From Fig.3d, it is shown that the square and circle shapes give the least blocking probability, while it is shown from Fig. 3e.All shapes are roughly the same with a little privilege for triangle in congestion period, whilst circle and triangle shapes give the least blocking probability as shown in Fig. 3f.

From above, the triangle and the circle models can be taken as the best shape for outer cell according to the blocking probability of the outer users.

Fig. 4 ( $a$ and $b$ ) shows the relation between blocking probability of the four models with time in all inner and outer cells for entire system, respectively.

From Fig. 4a, it is shown that the triangle shape give the least blocking probability, while it is shown from Fig. $4 \mathrm{~b}$ that the triangle and circle shapes give the least blocking probability. Best shape can be concluded from Fig.4c that illustrates the relation between blocking probability for entire system of the four models with time. Triangle system model is the best system model that gives the least blocking probability and hence the best performance, that is because triangle shape has the least distance between users and base station that leads to increase in value of $G_{x y}$. Therefore SINR value increases according to equation (3). Consequently, the blocking probability decreases. Fig. 5 shows the relation between THR of the four models with time for entire system. From this figure, we conclude that the triangle shape gives the max THR value.

\subsection{Impact of the Inner-Cell users Distribution}

Changing user distribution pattern in the inner-cell from fixed distribution into random distribution using the optimal design is discussed. It is cleared from Fig. 6 that better performance is obtained with fixed distribution than random distribution. That is because the maximum to average distance values for fixed distribution are less than random that leads to increase in both values of $G_{x y}$ and SINR. Consequently, blocking probability decreases.

\subsection{Impact of the Outer/Inner Cell-users \\ Ratio}

Using the best inner-cell shape (triangle), the impact of changing number of outer-cell users to the number of innercell users is introduced in Fig. 7. It is cleared that blocking probability decreases by decreasing the outer-cell users number (with a fixed inner cell users number), that is because as illustrated above that the maximum to average distance values between users and base station decreases that leads to increase in SINR and consequently decreasing in blocking probability to a certain point then increasing affected by interference of increasing the users number. Moreover, the overall system performance decreases (high $\mathrm{Pb}$ ) with the increase of the outer cell users to the inner cell users. That is because of congestion and high interference. From Fig. 7, the best ratio between the outer/inner users is 1:3.

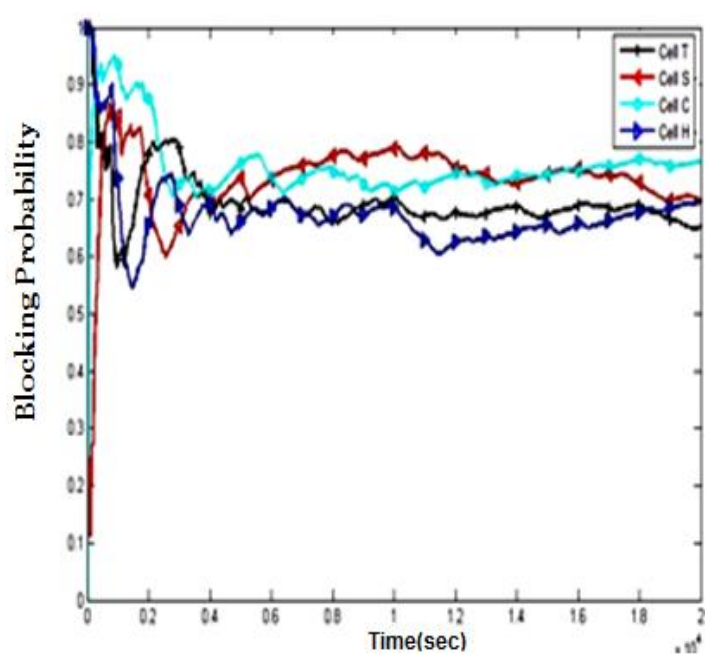

(3.a)

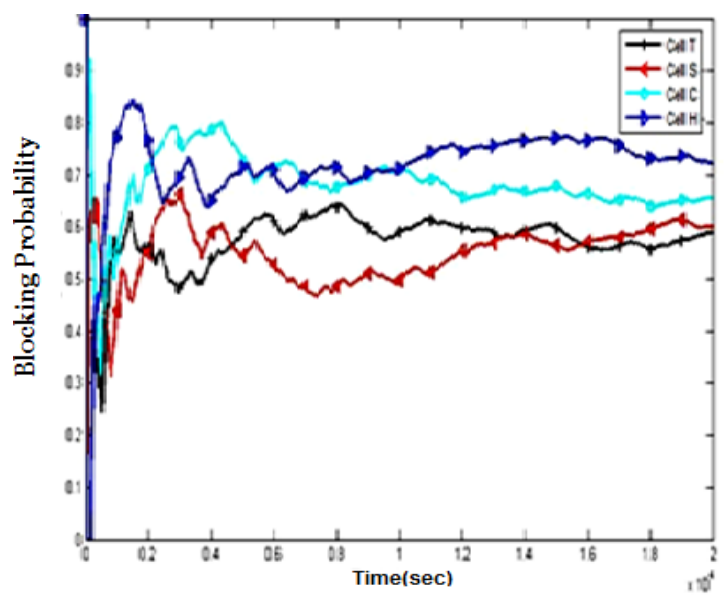

(3.b)

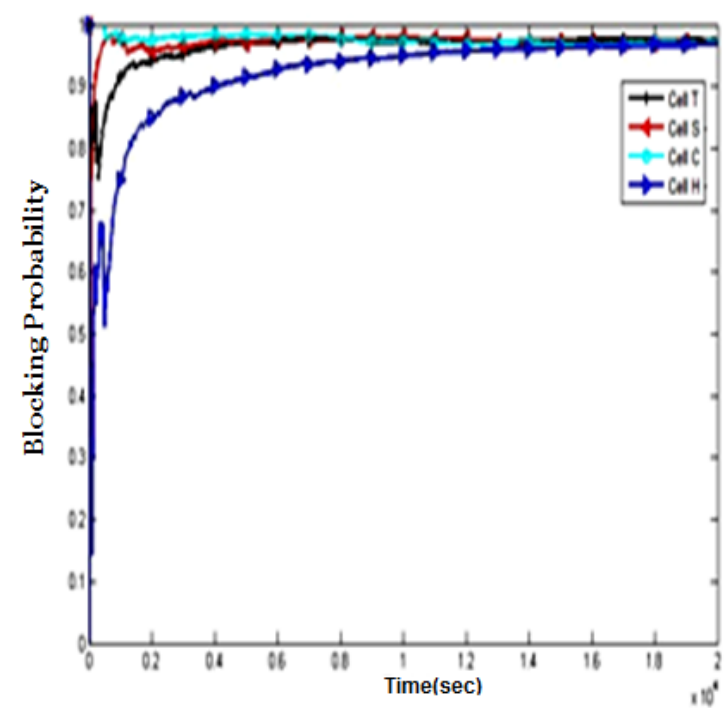

(3.c) 


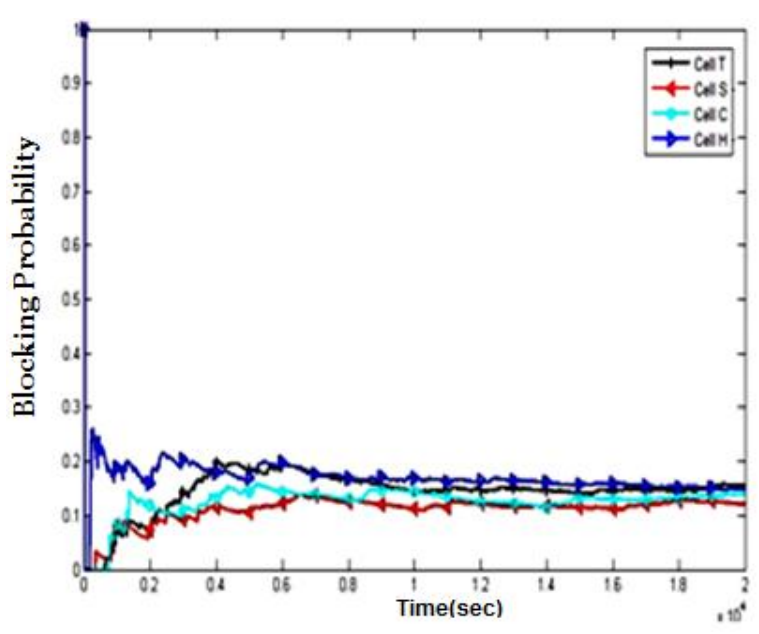

(3.d)

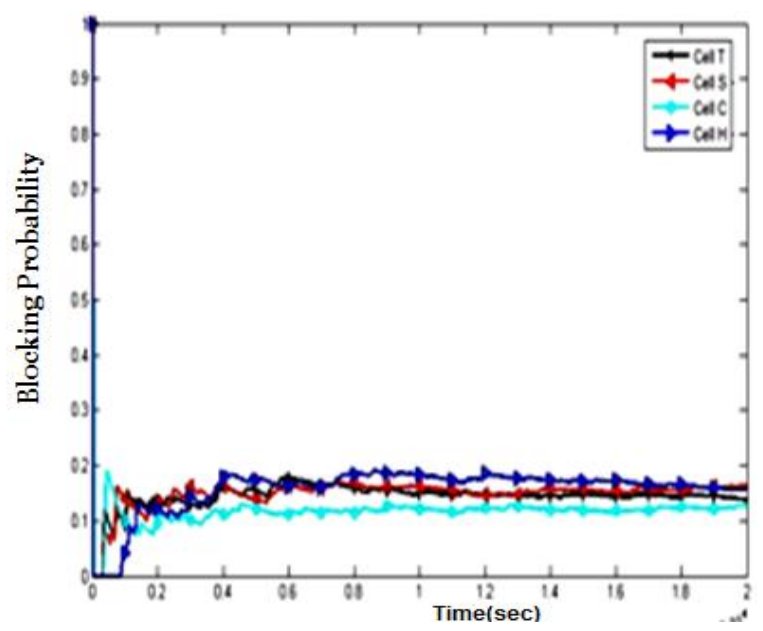

(3.e)

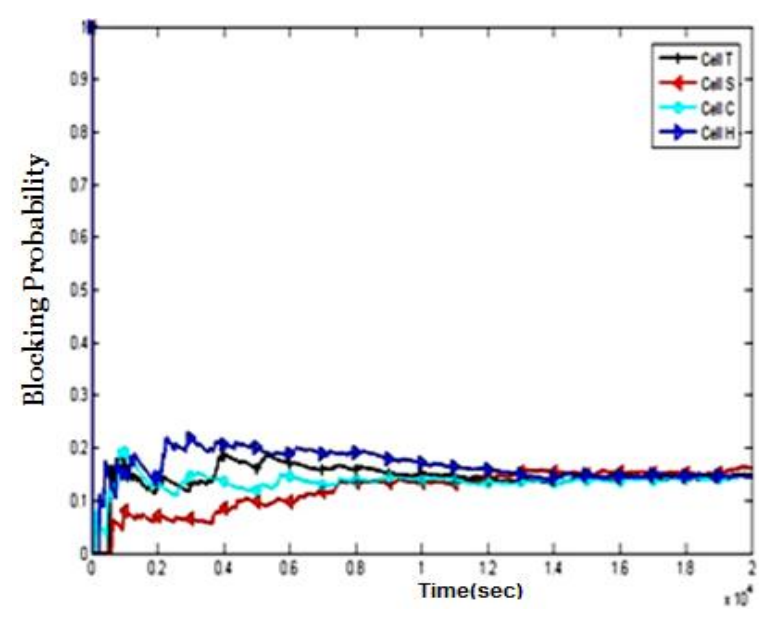

(3.f)

Fig.3 Relation between Blocking Probability \& time a)For inner cell 1. b)For inner cell 2. c) For inner cell 3. d)For outer cell 1. e)For outer cell 2. f)For outer cell 3.

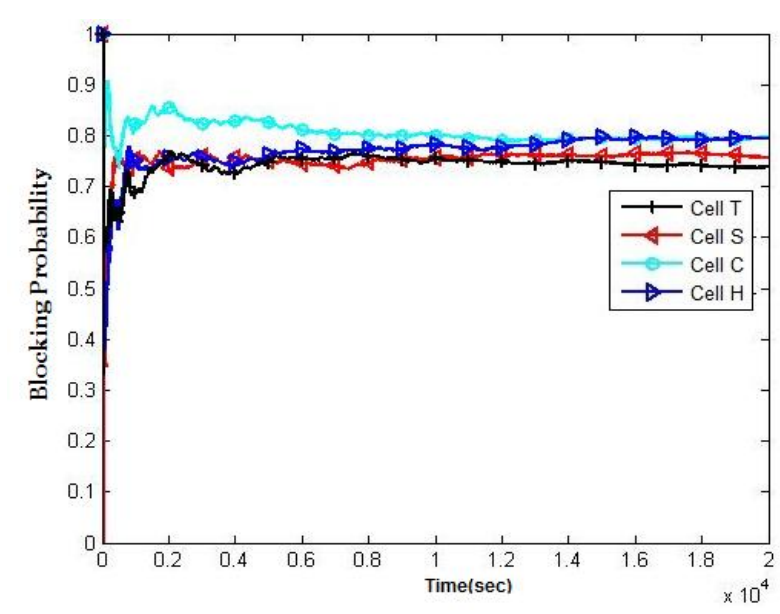

(4.a) For inner cells.

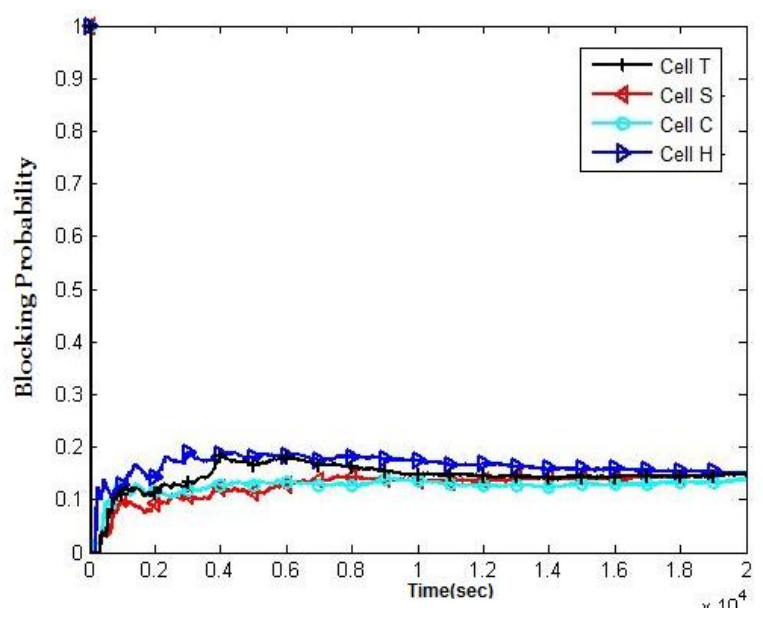

(4.b) For outer cells.

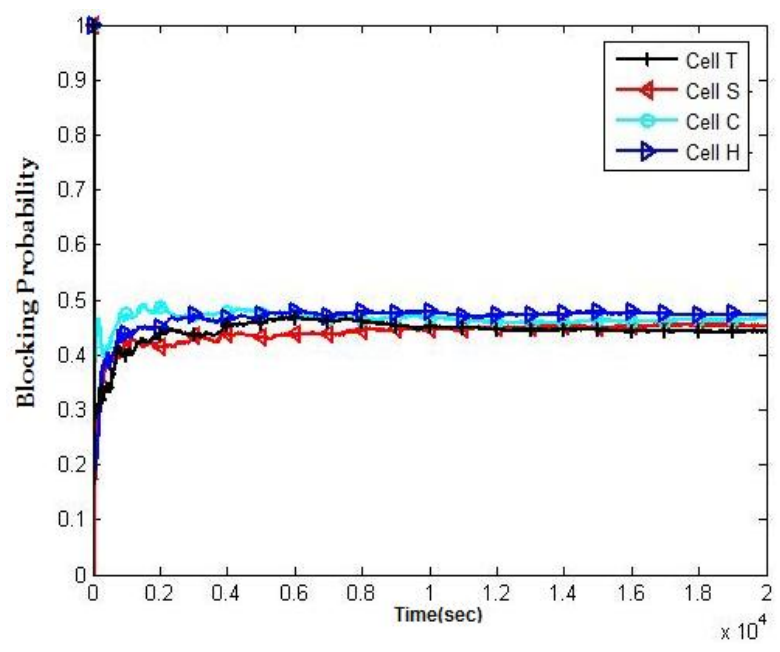

(4.c) For the entire system

Fig.4 Relation between Blocking Probability \&Time for all system models 


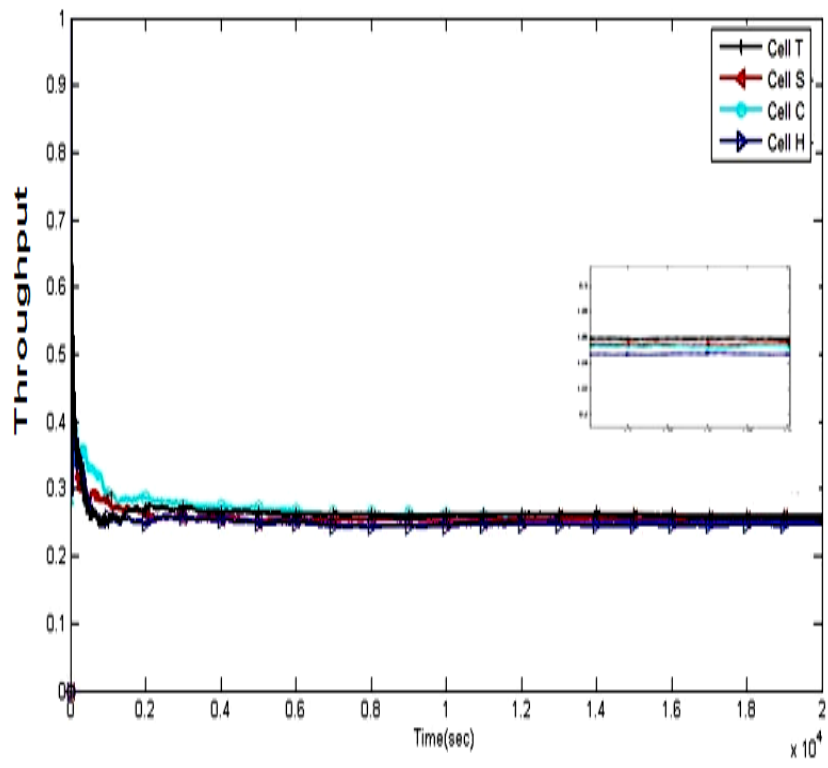

Fig.5 Relation between THR and system models

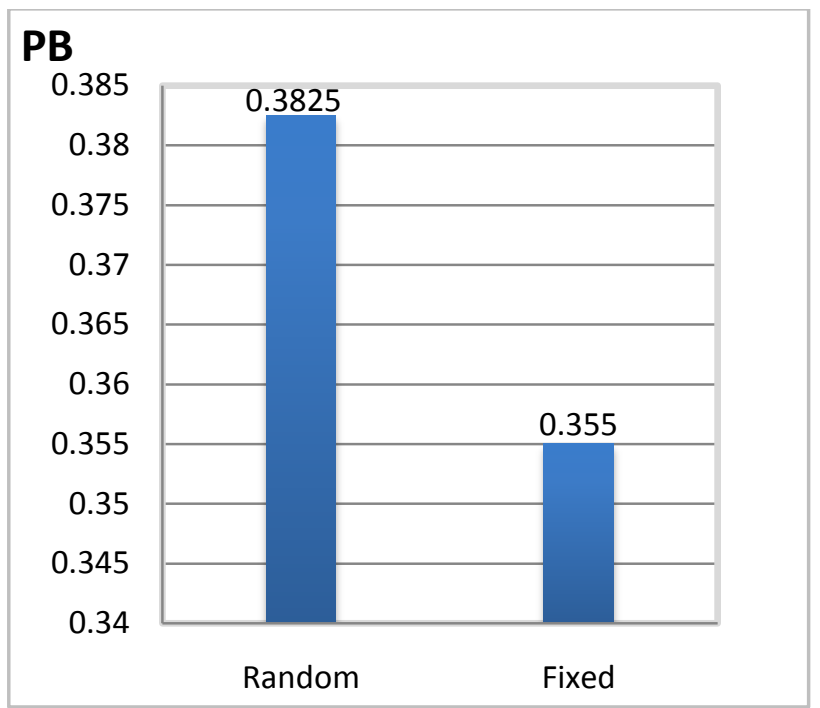

Fig. 6 Impact of changing inner-cell users distribution.

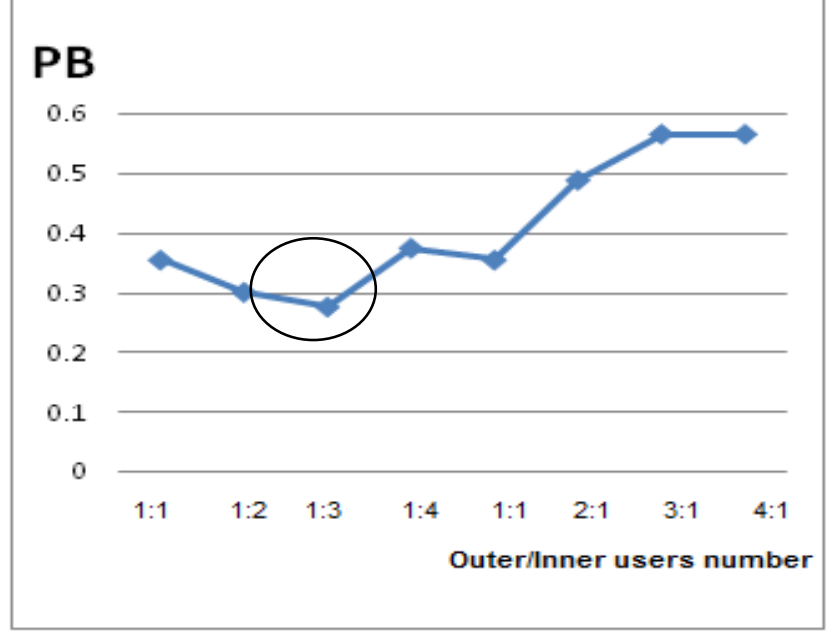

Fig. 7 Impact of the Outer/Inner Cell-users Ratio

\section{CONCLUTIONS}

In this paper, brief introduction about FFR schemes with focusing on strict FFR scheme is presented based on OFDMA systems. In addition, the best system model is selected to be triangle inner-cell shape when compared with three other different shapes. That is because it gives the minimum blocking probability and consequently, the best performance for entire system. Moreover, the impact of users' number and their distribution has introduced. The best system performance has been obtained when the ratio between the outer/inner users is minimized and the users are fixedly distributed, our future work will be based on introducing Cognitive Radio (CR) technique upon FFR schemes to increase system capacity and mitigate interference.

\section{REFRENCES}

[1] A.Racz, N.Reider and G.Fodor," On the Impact of InterCell Interference in LTE”, IEEE GLOBECOM, pp. 1-6, December 2008.

[2] L.Daewon, G.Y Li, and T.Suwen," Inter-cell interference coordination for LTE systems',IEEE GLOBECOM, pp. 4828-4833, December 2012.

[3] G.Boudreau, J.Panicker, N.Guo, R.Chang, N.Wang and S.Vrzic," Interference Coordination and Cancellation for 4G Networks", IEEE Communications Magazine, vol. 47, pp. 74-81, April 2009.

[4] Poongup Lee, "Interference Management in LTE Femtocell Systems Using Fractional frequency Reuse", Advanced Communication Technology (ICACT), $12^{\text {th }}$ International Conference, vol. 2, pp. 1047-1051, February 2010.

[5] N.Saquib, E.Hossam and D.IN Kim, "Fractional Frequency Reuse for Interference Management in LTEAdvanced Hetnets", Wireless Communication, IEEE, vol. 20, pp. 1536-1284, April 2013.

[6] T.D.Novlan, R.K.Ganti, A.Ghosh and G.J.Andrews, 'Analytical Evaluation of Fractional Frequency Reuse for OFDMA Cellular Networks'Wireless Communication, IEEE, vol. 10,pp. 4293-4305, October 2011.

[7] T.Novlan, J.G.Anderews, I.Sohn and R.K.Ganti,"Comparison of Fractional Frequency Reuse Approaches in the OFDMA Cellular Downlink", Global Telecommunications Conference (GLOBECOM) IEEE, pp. 1-5, December 2010.

[8] R.Chang, Z.Tao, J.Zhang and C.C.Kuo," A Graph Approach to Dynamic Fractional 'Frequency Reuse (FFR) in Multi-cell OFDMA Networks" IEEE International Conference, pp. 1-6, June 2009.

[9] M.Assad,"Optimal Fractional Frequency Reuse (FFR) in Multicellular ODMA System",IEEE Vechicular Technology Conference, pp. 1-5, September 2008.

[10] M.Assad and N.Hassan,"Optimal Fractional Frequency Reuce (FFR) and Resource Allocation in Multiuser OFDMA System", International Conference on Information and Communication Technologies, pp. 8892, August 2009.

[11] Z.Bharucha and H.Haas," The distribution of path losses for uniformly distributed nodes in a circle", Research Letters in Communications, pp. 1-4, January 2008. 